\title{
Selective Nuclear Transparency Induced by Chaotic Scattering
}

\author{
C. H. Dasso, ${ }^{1}$ M. Gallardo, ${ }^{2}$ and M. Saraceno ${ }^{3}$ \\ ${ }^{1}$ The Niels Bohr Institute, University of Copenhagen, Blegdamsvej 17, DK-2100 Copenhagen Ø, Denmark \\ ${ }^{2}$ Departamento de Física Atómica, Molecular y Nuclear, Universidad de Sevilla, Apdo. 1065, E-41080 Sevilla, Spain \\ ${ }^{3}$ Departamento de Física, Comisión Nacional de Energía Atómica, Av. del Libertador 8250, Buenos Aires, Argentina
}

(Received 15 November 1995)

\begin{abstract}
We investigate the effects of nuclear absorption on the population of intrinsic vibrational states in scattering processes involving heavy ions. It is found that at the classical level the removal of flux assumes a qualitatively different form in the regular or chaotic regimes. Specifically, it is shown how certain reaction channels turn out to be-in the latter case - relatively insensitive to the strength of the absorptive potential. This realization has a counterpart at the quantum level where, at the chaotic layer, conditions of selective transparency in the nuclear system are enforced. [S0031-9007(96)01465-2]
\end{abstract}

PACS numbers: 25.70.-z, 24.10.Eq, 24.60.Lz

It has been well established in a variety of calculations [1-3] that the coupling of the relative motion between two colliding ions to intrinsic collective modes of vibrational character leads to the occurrence of chaotic motion in the vicinity of the Coulomb and centrifugal potential barriers. The origin of this behavior can be traced to the existence of a separatrix in the classical phase space associated with the variables of relative motion and the unstable hyperbolic point that sits at its crossing at the top of the barrier. As the coupling to the intrinsic degrees of freedom is switched on, the formerly sharp separatrix develops into a chaotic layer whose physical extent is a measure of the size of the deformation parameters involved. Similar conclusions have also been reached in reactions involving statically deformed nuclei [4,5], recently reviewed in Ref. [6]. For a general introduction to the subject of chaotic scattering, see, e.g., Ref. [7].

Classical chaotic motion results from the fact that trajectories that go over the barrier are allowed to explore the potential pocket and return to the vicinity of the hyperbolic point. The presence of a homoclinic intersection in the nuclear interior is sufficient to alter the organization of the phase space and yield the rich and complex structures that characterize the irregular regime. Clearly, the probability of remaining within the space of explicitly-treated reaction channels diminishes rapidly for those orbits where the relative motion is entertained for an appreciable extent of time inside the strong interaction radius. The actual manifestation of the irregular motion at both the classical and quantum levels thus relies strongly on conditions of weak absorption [1]. Under these conditions the usual sharp distinction between above and below barrier phenomena becomes blurred and an energy "window" appears which is characterized classically by irregular deflection and reaction functions and quantally by the appearance of fluctuations in cross sections and angular distributions.

In this Letter we investigate the way in which the strength of an absorptive potential affects the population of states of intrinsic motion in regular and irregular situations. Following the understanding derived from regular situations, one could think that a growing strength in the absorptive potential would remove flux more or less uniformly from all channels above the barrier while leaving quite unaffected those below. We show that this is not the case and that in the chaotic layer the absorption has very irregular effects on the population of states. This behavior has the remarkable consequence of "shielding" the absorption for specific channels and therefore enforcing a selective transparency of the nuclear system.

To illustrate this phenomenon we shall use in this communication the same model introduced in Ref. [1]. The number of degrees of freedom of a heavy ion scattering problem is there reduced to a minimum without sacrificing the basic elements that are needed to investigate the coexistence of regular and irregular motion at the actual nuclear scales. We consider coupling to a harmonic mode through a Hamiltonian of the form

$$
\begin{aligned}
H_{\ell}(r, p, \alpha, \Pi)= & \frac{p^{2}}{2 m}+\frac{\ell(\ell+1) \hbar^{2}}{2 m r^{2}}+V(r) \\
& +\frac{C \alpha^{2}}{2}+\frac{\Pi^{2}}{2 D}+R_{0} \frac{\partial V_{N}}{\partial r} \alpha,
\end{aligned}
$$

where $r$ is the distance between the centers of mass of the colliding systems and $\alpha$ is the dimensionless variable that measures the amplitude of the vibrational motion. The variables $p, \Pi$ are, respectively, their conjugate momenta. We use $m$ for the reduced mass and $C$ and $D$ for the restoring force and mass parameters associated with the mode. $R_{0}$ is the equilibrium radius of the vibrating system. The real potential $V(r)$ represents the combined effects of the Coulomb interaction and of the nuclear potential $V_{N}(r)$, which we take here to be a Woods-Saxon version of the Christensen-Winther empirical potential of Ref. [8].

We solve the classical equations of motion derived from the previous Hamiltonian for a given combination of projectile and target choosing $C$ and $D$ from characteristic values of the deformation parameters $\beta$ and excitation energy of the mode $\hbar \omega$. The relationship between these parameters is given in Eq. (3) of Ref. [1]. In 
what follows we take-just to define a nuclear scalea head-on collision between two calcium nuclei and an intrinsic excitation energy $\hbar \omega=2 \mathrm{MeV}$. The value of $\beta$ we reserve as our parameter to control the extent of the chaotic region that develops in the vicinity of the Coulomb barrier.

We first look at the classical aspects. One of the most convenient ways to display the onset of irregular motion is by using reaction functions that correlate the final value of an observable with a classical variable which remains unspecified in the initial quantal state. In our problem only the initial energy of intrinsic motion is given, for instance $E_{\text {int }}(t=0)=\frac{1}{2} \hbar \omega$ (corresponding to $n_{i}=0$ ), and one plots the final "classical" number of phonons, defined as $n_{f}=E_{\text {int }}(t=\infty) / \hbar \omega-\frac{1}{2}$ for a uniform sample of all possible initial values of the actionangle conjugate variable $\varphi_{i}$.

The main features of the irregular window that opens around the barrier can be appreciated in Fig. 1, where the final number of phonons is displayed as a function of the variable $\varphi_{i}$ and the bombarding energy $E$. The shades of grey indicate the values of the reaction function $n_{f}$ (white is higher), and the two frames are for two different values of $\beta$. One can clearly follow in the figure the growth of the chaotic layer for energies around the Coulomb barrier $V_{B} \approx 56 \mathrm{MeV}$. Well below and well above the barrier there are regions where the reaction function is regular. They involve trajectories that change smoothly with energy and initial phase angle and that have only one turning point in the radial coordinate. In between, different families of trajectories of increasing complexity are responsible for the rich structures exhibited there.

The problem posed above is solved in the context of quantum mechanics within a coupled-channel framework that makes use of the basis of asymptotic channels $H_{\text {int }}|n\rangle=\left(n+\frac{1}{2}\right) h \omega|n\rangle$ of the intrinsic harmonic mode. The removal of flux is here introduced by adding an imaginary component to the nuclear potential, namely

$$
V_{N}(r) \rightarrow U_{N}(r)=V_{N}(r)+i W(r) .
$$

The classical counterpart to this procedure for a weak absorption results from ascribing to each trajectory a survival probability $\rho$, defined as

$$
\rho=\exp \left[\frac{2}{\hbar} \int_{0}^{\infty} W(r(t)) d t\right],
$$

where the integration is carried along the trajectory [9]. When this factor is taken into account the different trajectories that contribute to the scattering are selectively weighted according to the length of the path inside the potential, effectively cutting off the more complicated ones. The distribution of classical probabilities $P(n)-$ namely the probability that the process leads to the

$$
\beta=0.01
$$

$$
\beta=0.04
$$
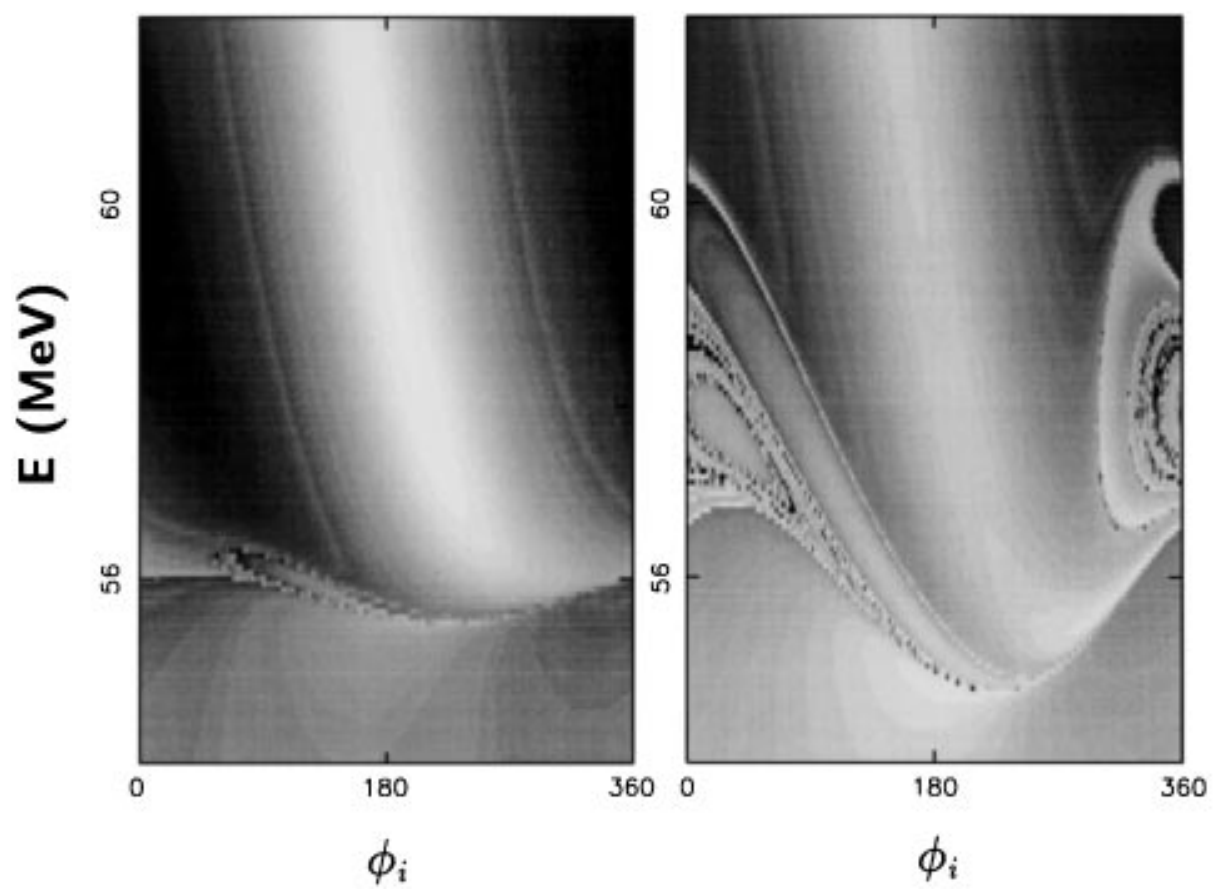

FIG. 1. Contours of the excitation function $n_{v}\left(E_{\mathrm{tot}}, \phi_{i}\right)$, i.e., the final number of phonons as a function of total energy and initial orientation angle in the intrinsic $\alpha \Pi$ plane. The calculations are for nuclear scales defined by a collision between calcium nuclei and an intrinsic excitation energy $\hbar \omega=2 \mathrm{MeV}$. The vibrational mode is originally in its ground state $\left(n_{i}=0\right)$ and the two frames correspond to deformation parameters $\beta=0.01$ (left) and $\beta=0.04$ (right). 
excitation of a number of phonons $n$-is constructed from a large ensemble of trajectories calculated for a set of initial angles $\varphi_{i}$, as described in [1]. This is done by projecting the reaction function $n_{f}\left(\varphi_{i}\right)$ in a regular grid $n$ so that $n \leq n_{f} \leq n+\Delta n$. The resulting probability distributions are stable provided a sufficiently large number of trajectories feed the different bins of size $\Delta n$. This is not only true in the regular case (obvious) but also in the chaotic regime.

We show in Fig. 2 classical distribution of probabilities for the final number of phonons in a regular and irregular situation. The calculations have been made with an imaginary potential of a Woods-Saxon shape, $W(r)=$ $-W_{0} /\left\{1+\exp \left[\left(r-R_{W}\right) / a_{W}\right]\right\}$, where values of $R_{W}=$ $4 \mathrm{fm}, a_{W}=1 \mathrm{fm}$ have been chosen so that the absorption acts effectively inside of the potential pocket but are not otherwise critical. With a given geometry the strength of the absorption is then measured by the parameter $W_{0}$ and the three frames for the regular and irregular regimes correspond to $W_{0}=0,1$, and $5 \mathrm{MeV}$.

There is a qualitative difference between the two regimes. In the regular case the attenuation of probability occurs evenly over the entire range of final values of $n$. In the chaotic situation, however, the absorption does not affect all values of $n$ in a uniform way. There are reaction channels that are more attenuated than others. One can understand the origin of this different behavior.

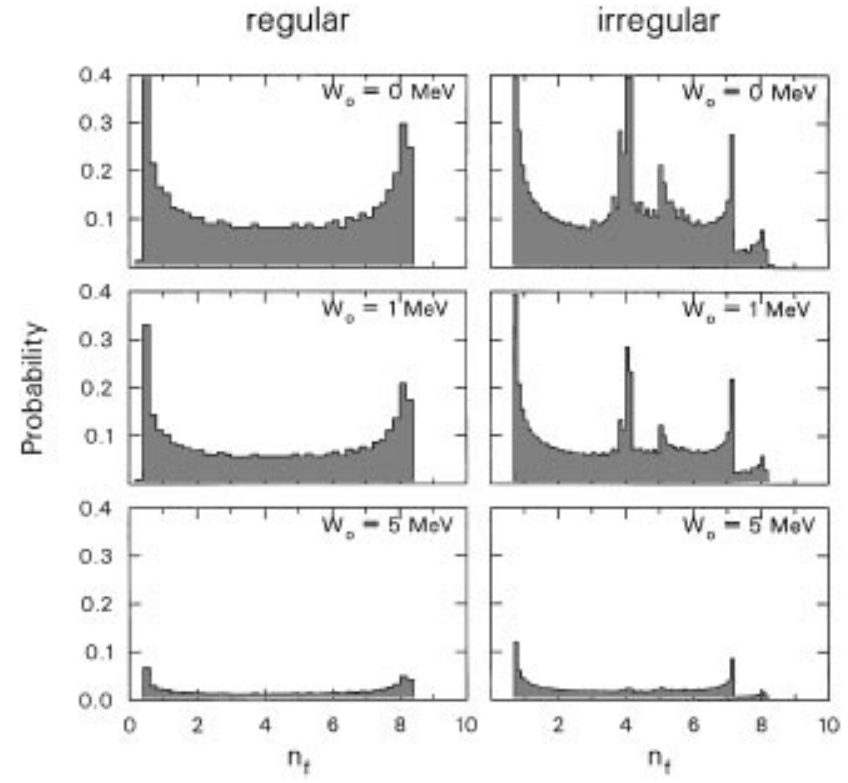

FIG. 2. Classical probability distributions $P\left(n_{f}\right)$ for a regular (left) and irregular (right) situation, and for three values of the absorptive potential strength $W_{0}$. These are constructed for $n_{i}=4$ by projecting the excitation functions $n_{f}\left(\phi_{i}\right)$ as described in Ref. [1]. The nuclear scales have been set as described in the caption to Fig. 1. The regular case corresponds to a bombarding energy $E_{\mathrm{tot}}=90 \mathrm{MeV}$ and a value of $\beta=$ 0.04 , while to illustrate the chaotic situation values $E_{\text {tot }}=$ $65 \mathrm{MeV}, \beta=0.035$ have been selected.
Indeed - and as it was anticipated above - the entire set of trajectories associated with the regular regime is of a similar nature and therefore the survival probability (3) has a smooth and slowly-varying behavior in the whole regular region. In the chaotic zone, on the other hand, the reaction function is broken up in many distinct domains that correspond to families of orbits which have an increasing degree of complexity. Naturally, these trajectories are absorbed at different rates for the same value of $W_{0}$, and this is reflected in Fig. 2.

We are not considering here the interference between different trajectories leading to the same final values of $n_{f}$. Therefore one should not expect to reproduce the oscillating patterns that characterize the quantal probabilities. This dimension of the problem is very interesting especially in the chaotic regime where exponentially many trajectories interfere-but not essential to the shielding effects revealed here and will be treated in detail elsewhere.

Since the coupled-channel solutions involve just an integration of the Schrödinger equation along the radial coordinate in the presence of $W(r)$ it is hard to visualize a mechanism that could lead to different rates of attenuation for the intrinsic states in the chaotic regime, as suggested by the classical results. Figure 3 shows, however, that this is indeed the case. To the left of the figure we show the excitation probabilities $P(n)$ as a function of $W_{0}$ obtained in a regular situation. All the reaction channels are attenuated evenly, as intuitively expected. At the right-hand-side the probabilities $P(n)$ are displayed for a chaotic case, and it is there obvious that the effects of the imaginary potential are felt unevenly. Specifically, there is a number of channels which appear relatively immune to the strength of the absorption. We note that both types of behavior appear intertwined in the channel label, i.e., do not follow the natural order prescribed by the intrinsic excitation energies.
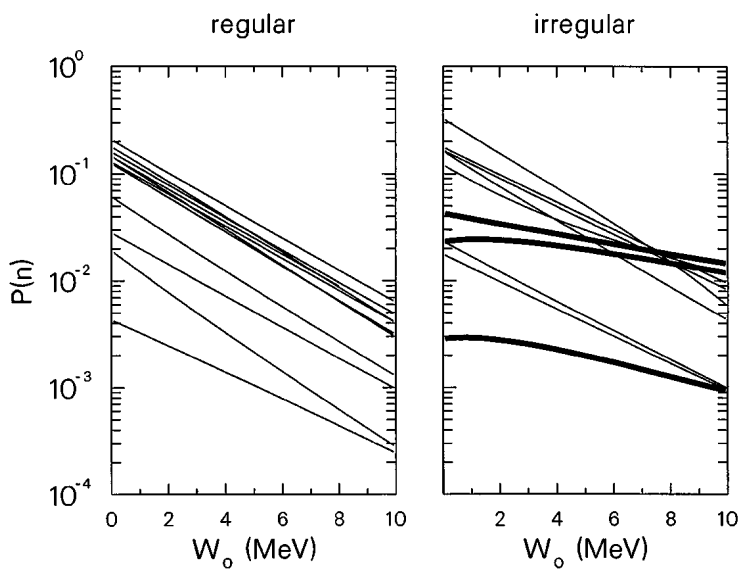

FIG. 3. Quantum mechanical probabilities $P(n)$ as a function of the strength $W_{0}$ of the absorptive potential, resulting from coupled-channel calculations for $E_{\text {tot }}=85 \mathrm{MeV}, n_{i}=3$, and $E_{\mathrm{tot}}=70 \mathrm{MeV}, n_{i}=4$ that correspond, respectively, to classical regular and irregular situations. 
A plausible explanation of the observed effects is that the wave functions for the channels that are not affected by the absorption are kept outside of the nuclear pocket and therefore "protected" from the flux removal that inevitably occurs when wave functions and imaginary potentials overlap. This is in fact what happens in the case of resonant chaotic scattering, as pointed out in Ref. [2] (cf. e.g., Fig. 8 of [2]). Such effects could also be related to a general phenomenon observed in the eigenfunctions of many bound chaotic systems which exhibit so-called "scars" [10]. These are regions of phase space close to unstable periodic orbits where the amplitude of the eigenfunctions is abnormally high. We have indeed found in our calculations scattering states strongly localized at the barrier and with a very small amplitude inside the potential pocket. Because of the very complex structure of the multistep feeding patterns, however, a clear relationship between this feature and the enhanced transparency cannot be easily established.

The results presented here are important because they point towards a better chance of observing signatures of chaotic scattering under the conditions that prevail in actual nuclear systems, namely, those of strong absorption inside the touching radii. The difficulty to visualize the mechanisms that produce these unfamiliar results underscores, on the other hand, the fundamental difference that exists between the regular and irregular regimes insofar as intuition is concerned. As we have stressed earlier [3], successful semiclassical modeling relies - to a degree that one is often unaware of - on the fact that the quantization of states may still take place on tori, even in the presence of coupling. When this is not the case, there are no clear guidelines to relate the properties of the eigenstates of the combined system to the asymptotic states which are labeled by the quantum numbers of intrinsic and relative motion and which are the source of qualitative understanding in many instances.

In conclusion, both the classical and the quantum calculations show that absorption acts in a radically different way in the regular and chaotic regimes. The latter case is characterized by a strong dependence on channel number and energy. The main ideas were developed and illustrated within a simplified reaction model. Inasmuch as they are related to general issues of chaotic behavior the features revealed here will, nonetheless, remain present in more complicated coupling schemes. Indeed, preliminary studies [11] show that it is possible to extract quantum reaction and deflection functions from realistic, state-of-the-art coupled-channel codes like FRESCO [12], and that many aspects of the results are closely correlated with classical trajectories, both regular and chaotic. It is also interesting to point out that our model calculations clearly indicated that numerical integration of the coupled-channel equations in the chaotic regime requires a much higher level of accuracy and care than in the regular case. This has been observed also in the aforementioned full quantal calculations, where the irregular dependence of the population patterns as a function of an increased absorption appears to be one of the criteria that can be used to determine the chaotic character of the motion even when a classicallyequivalent model is not available.

This work was supported in part by Grant No. DFG Bo 1109/1, the Heraeus Foundation No. CONYCET Pid. 3233/92, the Fundación Antorchas, Spanish CICYT Project No. PB95-0533-A, and EU Network Contract No. CHRX-CT92-0075.

[1] C. H. Dasso, M. Gallardo, and M. Saraceno, Nucl. Phys. A459, 265 (1992).

[2] C. H. Dasso, M. Gallardo, and M. Saraceno, Nucl. Phys. A587, 339 (1995).

[3] C. H. Dasso, M. Gallardo, and M. Saraceno, in Heavy Ion Fusion; Exploring the Variety of Nuclear Properties, edited by A. Stefanini et al. (World Scientific Publishing Co., Singapore, 1995), p. 1; Rev. Bras. Fis. 24, 643 (1994); in Proceedings of the 1994 Summer School in La Rabida, Spain, J. Arias et al. (Springer Verlag, Berlin, 1995), p. 231.

[4] A. Rapisarda and M. Baldo, Phys. Rev. Lett. 66, 2581 (1991).

[5] M. Baldo, E. G. Lanza, and A. Rapisarda, Nucl. Phys. A545, 467c (1992).

[6] M. Baldo, E. G. Lanza, and A. Rapisarda, Chaos 3, 691 (1993).

[7] U. Smilansky, in Chaos and Quantum Physics, edited by M. Giannoni, A. Voros, and J. Zinn-Justin (North-Holland, Amsterdam, 1991).

[8] P. R. Christensen and A. Winther, Phys. Lett. B 65, 19 (1978).

[9] R. Broglia and A. Winther, Heavy Ion Reactions (Addison-Wesley Publishing Company, Redwood City, CA, 1991).

[10] R. V. Jensen, M. M. Sanders, M. Saraceno, and B. Sundaran, Phys. Rev. Lett. 63, 2771 (1989).

[11] C. H. Dasso, G. Pollarolo, and M. Saraceno, Nucl. Phys. A602, 77 (1996).

[12] I. J. Thompson, Comput. Phys. Rep. 7, 167 (1988). 